\title{
Existence of Renormalized Solution of Some Elliptic Problems in Orlicz Spaces
}

\author{
Lahsen Aharouch, Jaouad BennounA, \\ and Abdelfettah TOUZANI
}

\author{
Faculté polydisciplinaire Ouarzazate \\ BP 638 Ouarzazate - Morocco \\ laharouch@gmail.com
}

\author{
Département de Mathématiques et Informatique \\ Faculté des Sciences Dhar-Mahraz \\ B.P 1796 Atlas Fès - Morocco \\ jbennouna@hotmail.com atouzani@menara.ma
}

Received: January 18, 2007

Accepted: January 24, 2008

\section{ABSTRACT}

In this paper, we study the problem

$$
-\operatorname{div} a(x, u, \nabla u)-\operatorname{div} \phi(u)+g(x, u)=f
$$

in the framework of Orlicz spaces. The main contribution of our work is to prove the existence of a renormalized solution without any restriction on the $N$-function of the Orlicz space.

Key words: Orlicz Sobolev spaces, boundary value problems, truncations, renormalized solutions.

2000 Mathematics Subject Classification: 35J60.

\section{Introduction}

Let $\Omega$ be a bounded open subset of $\mathbb{R}^{N}$ and let $A u=-\operatorname{div} a(x, u, \nabla u)$ be a LerayLions operator defined in $W_{0}^{1, p}(\Omega), 1<p<\infty$.

We consider the following nonlinear elliptic problem:

$$
\left\{\begin{aligned}
-\operatorname{div} a(x, u, \nabla u)-\operatorname{div} \phi(u)+g(x, u)=f & \text { in } \quad \Omega \\
u=0 & \text { on } \quad \partial \Omega
\end{aligned}\right.
$$


where $f \in W^{-1, p^{\prime}}(\Omega), \phi \in \mathcal{C}^{0}\left(\mathbb{R}, \mathbb{R}^{N}\right)$, and $g$ is a Carathéodory function satisfying

$$
\sup _{|t| \leq n}|g(\cdot, s)|=h_{n}(\cdot) \in L^{1}(\Omega) \quad \forall n .
$$

Note that no growth hypothesis is assumed on the function $\phi$ which implies that the term $\operatorname{div} \phi(u)$ may be meaningless, even as a distribution. The notion of renormalized solution (see definition 2.1) gives a meaning to a possible solution of (1).

In the case where $\phi=0$, existence of a weak solution in the usual sense to (1) is proved by Rakotoson and Temam [16].

The notion of renormalized solutions in the usual case was introduced by R. J. DiPerna and P.-L. Lions [10] for the study of the Boltzmann equations. This notion was then adapted to the study of the problem (1) by L. Boccardo et al. [8] when the right hand side is in $W^{-1, p^{\prime}}(\Omega)$, by J. M. Rakotoson [15] when the right hand side is in $L^{1}(\Omega)$, and finally by G. Dal Maso et al. [9] for the case in which the right hand side is general measure data.

The functional setting in these works is the usual Sobolev space $W^{1, p}(\Omega)$. Accordingly the function $a(\cdot)$ is supposed to satisfy polynomial growth conditions with respect to $u$ and its derivatives $\nabla u$.

When trying to perform an analysis for the function $a(\cdot)$ with more general growth conditions, one is led to replace $W^{1, p}$ by a Sobolev-space $W^{1} L_{M}$ built from an Orlicz space $L_{M}$ instead of $L^{p}$. Here the $N$-function $M$ which defines $L_{M}$ is related to the actual growth of the function $a$

Recently Benkirane and Bennouna [5] have generalized the last result of Boccardo et al. [8] to the Orlicz-Sobolev space with some restrictions on the $N$-function (i.e., the $\Delta_{2}$-condition).

It is our purpose, in this paper, to prove the existence of renormalized solution for the problem (1) in the setting of the Orlicz Sobolev space $W_{0}^{1} L_{M}(\Omega)$ without any restriction on the $N$-function $M$. (i.e., without the $\Delta_{2}$-condition). See theorem 2.3. This paper is organized as follows: Section 1 contains some preliminaries and some technical lemmas concerning convergence in Orlicz Sobolev space. In section 2, we state our main result which will be proved in section 3. The proof uses techniques different from that given in $[5,8]$.

For some existence results for strongly non-linear elliptic equation in Orlicz space see $[2-4,6]$

\section{Preliminaries}

\subsection{N-function}

Let $M: \mathbb{R}^{+} \rightarrow \mathbb{R}^{+}$be an $N$-function, i.e., $M$ is continuous, convex, with $M(t)>0$ for $t>0, \frac{M(t)}{t} \rightarrow 0$ as $t \rightarrow 0$ and $\frac{M(t)}{t} \rightarrow \infty$ as $t \rightarrow \infty$. Equivalently, $M$ admits the representation $M(t)=\int_{0}^{t} a(s) d s$, where $a: \mathbb{R}^{+} \rightarrow \mathbb{R}^{+}$is a nondecreasing, right continuous function, with $a(0)=0, a(t)>0$ for $t>0$, and $a(t)$ tends to $\infty$ as $t \rightarrow \infty$. 
The $N$-function $\bar{M}$, conjugate to $M$, is defined by $\bar{M}(t)=\int_{0}^{t} \bar{a}(s) d s$, where $\bar{a}: \mathbb{R}^{+} \rightarrow \mathbb{R}^{+}$is given by $\bar{a}(t)=\sup \{s: a(s) \leq t\}$.

The $N$-function $M$ is said to satisfy the $\Delta_{2}$-condition if for some $k$

$$
M(2 t) \leq k M(t) \quad \forall t \geq 0 .
$$

It is readily seen that this will be the case if and only if for every $r>0$ there exists a positive constant $k=k(r)$ such that for all $t>0$

$$
M(r t) \leq k M(t) \quad \forall t \geq 0 .
$$

When (2) and (3) hold only for $t \geq t_{0}$, for some $t_{0}>0$, then $M$ is said to satisfy the $\Delta_{2}$-condition near infinity.

We will extend these $N$-functions into even functions on all $\mathbb{R}$. Moreover, we have the following Young's inequality:

$$
\forall s, t \geq 0, \quad s t \leq M(t)+\bar{M}(s) .
$$

Let $P$ and $Q$ be two $N$-functions. We say that $P$ grows essentially less rapidly than $Q$ near infinity, and denote it $P \ll Q$, if for every $\varepsilon>0, \frac{P(t)}{Q(\epsilon t)} \rightarrow 0$ as $t \rightarrow \infty$. This is the case if and only if $\lim _{t \rightarrow \infty} \frac{Q^{-1}(t)}{P^{-1}(t)}=0$ (see [1, chapter 8]).

\subsection{Orlicz space $L_{M}(\Omega)$}

Let $M$ be an $N$-function and $\Omega \subset \mathbb{R}^{N}$ be an open and bounded set. The Orlicz class $\mathcal{K}_{M}(\Omega)$ (resp. the Orlicz space $L_{M}(\Omega)$ ) is defined as the set of (equivalence classes of) real valued measurable functions $u$ on $\Omega$ such that

$$
\left.\int_{\Omega} M(u(x)) d x<+\infty \quad \text { (resp. } \int_{\Omega} M\left(\frac{u(x)}{\lambda}\right) d x<+\infty \text { for some } \lambda>0\right) .
$$

$L_{M}(\Omega)$ is a Banach space under the norm,

$$
\|u\|_{M, \Omega}=\inf \left\{\lambda>0: \int_{\Omega} M\left(\frac{u(x)}{\lambda}\right) d x \leq 1\right\}
$$

and $\mathcal{K}_{M}(\Omega)$ is a convex subset of $L_{M}(\Omega)$ but not necessarily a linear space.

The closure in $L_{M}(\Omega)$ of the set of bounded measurable functions with compact support in $\bar{\Omega}$ is denoted by $E_{M}(\Omega)$.

The dual space of $E_{M}(\Omega)$ can be identified with $L_{\bar{M}}(\Omega)$ by means of the pairing $\int_{\Omega} u v d x$, and the dual norm of $L_{\bar{M}}(\Omega)$ is equivalent to $\|\cdot\|_{\bar{M}, \Omega}$.

Let $X$ and $Y$ be arbitrary Banach spaces with bilinear bicontinuous pairing $\langle,\rangle_{X, Y}$.

We say that a sequence $\left\{u_{n}\right\} \subset X$ converges to $u \in X$ with respect to the topology $\sigma(X, Y)$, denoted by $u_{n} \rightarrow u(\sigma(X, Y))$, in $X$, if $\left\langle u_{n}, v\right\rangle \rightarrow\langle u, v\rangle$ for all $v \in Y$. For example, if $X=L_{M}(\Omega)$ and $Y=L_{\bar{M}}(\Omega)$, then the pairing is defined by

$$
\langle u, v\rangle=\int_{\Omega} u(x) v(x) d x \quad \forall u \in X, v \in Y .
$$




\subsection{Orlicz-Sobolev space}

We now turn to the Orlicz-Sobolev space, $W^{1} L_{M}(\Omega)\left(\right.$ resp. $\left.W^{1} E_{M}(\Omega)\right)$ is the space of all functions $u$ such that $u$ and its distributional derivatives up to order 1 lies in $L_{M}(\Omega)\left(\right.$ resp. $\left.E_{M}(\Omega)\right)$. It is a Banach space under the norm

$$
\|u\|_{1, M}=\sum_{|\alpha| \leq 1}\left\|D^{\alpha} u\right\|_{M} .
$$

Thus, $W^{1} L_{M}(\Omega)$ and $W^{1} E_{M}(\Omega)$ can be identified with subspaces of product of $N+1$ copies of $L_{M}(\Omega)$. Denoting this product by $\prod L_{M}$, we will use the weak topologies $\sigma\left(\prod L_{M}, \prod E_{\bar{M}}\right)$ and $\sigma\left(\prod L_{M}, \prod L_{\bar{M}}\right)$.

The space $W_{0}^{1} E_{M}(\Omega)$ is defined as the (norm) closure of the Schwartz space $\mathcal{D}(\Omega)$ in $W^{1} E_{M}(\Omega)$ and the space $W_{0}^{1} L_{M}(\Omega)$ as the $\sigma\left(\prod L_{M}, \prod E_{\bar{M}}\right)$ closure of $\mathcal{D}(\Omega)$ in $W^{1} L_{M}(\Omega)$.

We say that a sequence $\left\{u_{n}\right\} \subset L_{M}(\Omega)$ converges to $u \in L_{M}(\Omega)$ in the modular sense, denoted $u_{n} \rightarrow u(\bmod )$ in $L_{M}(\Omega)$ if for some $\lambda>0$

$$
\int_{\Omega} M\left(\frac{\left|u_{n}(x)-u(x)\right|}{\lambda}\right) d x \longrightarrow 0 \quad \text { when } \quad n \rightarrow+\infty .
$$

We say that a sequence $\left\{u_{n}\right\} \subset W^{1} L_{M}(\Omega)$ converges to $u \in W^{1} L_{M}(\Omega)$ in the modular sense, denoted $u_{n} \rightarrow u(\mathrm{mod})$ in $W^{1} L_{M}(\Omega)$ if there exists $\lambda>0$ such that

$$
\int_{\Omega} M\left(\frac{\left|D^{\alpha} u_{n}(x)-D^{\alpha} u(x)\right|}{\lambda}\right) d x \longrightarrow 0 \quad \text { when } n \rightarrow+\infty \text { for all }|\alpha| \leq 1 .
$$

If $M$ satisfies the $\Delta_{2}$-condition (near infinity only when $\Omega$ has finite measure), then modular convergence coincides with norm convergence.

\subsection{Some lemmas}

Let $W^{-1} L_{\bar{M}}(\Omega)$ (resp. $W^{-1} E_{\bar{M}}(\Omega)$ ) denotes the space of distributions on $\Omega$ which can be written as sums of derivatives of order $\leq 1$ of functions in $L_{\bar{M}}(\Omega)$ (resp. $E_{\bar{M}}(\Omega)$ ). It is a Banach space under the usual quotient norm.

We recall some lemmas introduced in [7] which will be used later.

Lemma 1.1. Let $F: \mathbb{R} \rightarrow \mathbb{R}$ be uniformly Lipschitzian, with $F(0)=0$. Let $M$ be an $N$-function and let $u \in W^{1} L_{M}(\Omega)$ (resp. $W^{1} E_{M}(\Omega)$ ). Then $F(u) \in W^{1} L_{M}(\Omega)($ resp. $\left.W^{1} E_{M}(\Omega)\right)$. Moreover, we have

$$
\frac{\partial}{\partial x_{i}} F(u)=\left\{\begin{array}{lll}
F^{\prime}(u) \frac{\partial}{\partial x_{i}} u & \text { a.e. in } & \{x \in \Omega: u(x) \notin D\}, \\
0 & \text { a.e. in } & \{x \in \Omega: u(x) \in D\}
\end{array}\right.
$$

where $D$ is the set of discontinuity points of $F^{\prime}$. 
Lemma 1.2. Let $F: \mathbb{R} \rightarrow \mathbb{R}$ be uniformly Lipschitzian, with $F(0)=0$. Let $M$ be an $N$-function, then the mapping $T_{F}: W^{1} L_{M}(\Omega) \rightarrow W^{1} L_{M}(\Omega)$ defined by $T_{F}(u)=F(u)$ is sequentially continuous with respect to the weak* topology $\sigma\left(\prod L_{M}, \prod E_{\bar{M}}\right)$.

We give now the following lemma which concerns operators of the Nemytskii type in Orlicz spaces (see [7]).

Lemma 1.3. Let $\Omega$ be an open subset of $\mathbb{R}^{N}$ with finite measure. Let $M, P$, and $Q$ be $N$-functions such that $Q \ll P$, and let $f: \Omega \times \mathbb{R} \rightarrow \mathbb{R}$ be a Carathéodory function such that, for a.e. $x \in \Omega$ and all $s \in \mathbb{R}$,

$$
|f(x, s)| \leq c(x)+k_{1} P^{-1} M\left(k_{2}|s|\right),
$$

where $k_{1}, k_{2}$ are real constants and $c(x) \in E_{Q}(\Omega)$. Then the Nemytskii operator $N_{f}$ defined by $N_{f}(u)(x)=f(x, u(x))$ is strongly continuous from $\mathcal{P}\left(E_{M}(\Omega), \frac{1}{k_{2}}\right)=$ $\left\{u \in L_{M}(\Omega): d\left(u, E_{M}(\Omega)\right)<\frac{1}{k_{2}}\right\}$ into $E_{Q}(\Omega)$.

Below, we will use the following technical Lemmas.

Lemma $1.4([7])$. Let $\left(f_{n}\right), f, \gamma \in L^{1}(\Omega)$ such that

(i) $f_{n} \geq \gamma$ a.e. in $\Omega$,

(ii) $f_{n} \rightarrow f$ a.e. in $\Omega$,

(iii) $\int_{\Omega} f_{n}(x) d x \rightarrow \int_{\Omega} f(x) d x$.

Then $f_{n} \rightarrow f$ strongly in $L^{1}(\Omega)$.

We now turn to the approximation by functions which are smooth up to the boundary, assuming some regularity on $\Omega$. Recall that $\Omega$ is said to have the (interior) segment property if there exist an open covering $\left\{U_{i}\right\}$ of $\bar{\Omega}$ and corresponding vectors $\left\{y_{i} \in \mathbb{R}^{N}\right\}$ such that, for $x \in \bar{\Omega} \cap U_{i}$ and $0<t<1$, it is $x+t y_{i} \in \Omega$.

Lemma $1.5([12])$. Let $\Omega$ have the segment property. Then for each $\nu \in W_{0}^{1} L_{M}(\Omega)$, there exists a sequence $\nu_{n} \in \mathcal{D}(\Omega)$ such that $\nu_{n}$ converges to $\nu$ for the modular convergence in $W_{0}^{1} L_{M}(\Omega)$. Furthermore, if $\nu \in W_{0}^{1} L_{M}(\Omega) \cap L^{\infty}(\Omega)$ then

$$
\left\|\nu_{n}\right\| \leq(N+1)\|\nu\|_{L^{\infty}(\Omega)} .
$$

\section{Main result}

Let $\Omega$ be a bounded open subset of $\mathbb{R}^{N}$ satisfying the segment property. Let $A: \mathcal{D}(A) \subset$ $W_{0}^{1} L_{M}(\Omega) \rightarrow W^{-1} L_{\bar{M}}(\Omega)$ be a mapping given by $A(u)=-\operatorname{div} a(x, u, \nabla u)$, where $a$ is a function satisfying the following conditions:

$\left(A_{1}\right) a(x, s, \xi): \Omega \times \mathbb{R} \times \mathbb{R}^{N} \rightarrow \mathbb{R}^{N}$ is a Carathéodory function. 
$\left(A_{2}\right)$ There exist two $N$-functions $M$ and $P$ with $P \ll M$, a function $c(x)$ in $E_{\bar{M}}(\Omega)$, and positive constants $k_{1}, k_{2}, k_{3}, k_{4}$ such that

$$
|a(x, s, \zeta)| \leq c(x)+k_{1} \bar{P}^{-1} M\left(k_{2}|s|\right)+k_{3} \bar{M}^{-1} M\left(k_{4}|\zeta|\right),
$$

for a.e. $x$ in $\Omega$ and for all $s \in \mathbb{R}, \zeta \in \mathbb{R}^{N}$.

$\left(A_{3}\right)$ For a.e. $x$ in $\Omega, s \in \mathbb{R}$ and $\zeta, \zeta^{\prime}$ in $\mathbb{R}^{N}$, with $\zeta^{\prime} \neq \zeta$

$$
\left[a(x, s, \zeta)-a\left(x, s, \zeta^{\prime}\right)\right]\left(\zeta-\zeta^{\prime}\right)>0 .
$$

$\left(A_{4}\right)$ For a.e. $x$ in $\Omega, s \in \mathbb{R}$ and all $\zeta \in \mathbb{R}^{N}$,

$$
a(x, s, \zeta) \zeta \geq \alpha M\left(\frac{|\zeta|}{\lambda}\right)
$$

where $\alpha \in \mathbb{R}_{+}^{*}$.

Consider the nonlinear elliptic problem

$$
\left\{\begin{array}{rlr}
-\operatorname{div} a(x, u, \nabla u)-\operatorname{div} \phi(u)+g(x, u) & =f & \text { in } \quad \Omega, \\
u & =0 & \text { on } \quad \partial \Omega,
\end{array}\right.
$$

where

$$
f \in W^{-1} E_{\bar{M}}(\Omega),
$$

and $\phi=\left(\phi_{1}, \ldots, \phi_{N}\right)$ satisfy

$$
\phi \in\left(C^{0}(\mathbb{R})\right)^{N} .
$$

Let $g(x, t)$ be a Carathéodory function such that for a.e. $x \in \Omega$ and all $s \in \mathbb{R}$

$$
\begin{gathered}
g(x, s) s \geq 0, \\
\sup _{|t| \leq n}|g(\cdot, s)|=h_{n}(\cdot) \in L^{1}(\Omega) \quad \forall n .
\end{gathered}
$$

Note that no growth hypothesis is assumed on the function $\phi$, which implies that for a solution $u \in W_{0}^{1} L_{M}(\Omega)$ the term $\operatorname{div} \phi(u)$ may be meaningless, even as a distribution. As in [8] we define the following notion of renormalized solution, which gives a meaning to a possible solution of (4).

Definition 2.1. Assume that $\left(A_{1}\right)-\left(A_{4}\right),(5)-(8)$ hold true. A function $u$ is a renormalized solution of the problem (4) if

$$
\left\{\begin{array}{l}
u \in W_{0}^{1} L_{M}(\Omega), g(x, u) \in L^{1}(\Omega), \quad u g(x, u) \in L^{1}(\Omega) \\
-\operatorname{div} a(x, u, \nabla u) h(u)-\operatorname{div}(\phi(u) h(u))+\phi(u) h^{\prime}(u) \nabla u \\
+g(x, u) h(u)=f h(u) \operatorname{in} \mathcal{D}^{\prime}(\Omega), \quad \forall h \in \mathcal{C}_{c}^{1}\left(\mathbb{R}^{N}\right) .
\end{array}\right.
$$


The weaker problem (9) is obtained by using the test function $h(u)$ where $h \in \mathcal{C}_{c}^{1}(\mathbb{R})$ in $(4)$.

Remark 2.2. Let us note that in (9) every term is meaningful in the distributional sense.

It's easy to see that for $\varphi \in \mathcal{D}(\Omega)$ and $u \in W_{0}^{1} L_{M}(\Omega)$ we have $\varphi h(u) \in W_{0}^{1} L_{M}(\Omega)$ (one can apply Lemma 1.2) and

$$
\langle f h(u), \varphi\rangle_{\mathcal{D}^{\prime}(\Omega), \mathcal{D}(\Omega)}=\langle f, \varphi h(u)\rangle_{W^{-1} E_{\bar{M}}(\Omega), W_{0}^{1} L_{M}(\Omega)} .
$$

We have also $[-\operatorname{div} a(x, u, \nabla u)] \in W^{-1} L_{\bar{M}}(\Omega)$ and

$$
\begin{aligned}
\langle-\operatorname{div} a(x, u, \nabla u) h(u), \varphi\rangle_{\mathcal{D}^{\prime}(\Omega), \mathcal{D}(\Omega)} & \\
& =\langle-\operatorname{div} a(x, u, \nabla u), \varphi h(u)\rangle_{W^{-1} L_{\bar{M}}(\Omega), W_{0}^{1} L_{M}(\Omega)} .
\end{aligned}
$$

Finally since $\phi h$ and $\phi h^{\prime} \in\left(\mathcal{C}_{c}^{0}(\mathbb{R})\right)^{N}$ we have $\phi(u) h(u)$ and $\phi(u) h^{\prime}(u) \in\left(L^{\infty}(\Omega)\right)^{N}$, for any measurable function $u$ and then

$$
\operatorname{div}(\phi(u) h(u)) \in W^{-1, \infty}(\Omega), \quad \phi(u) h^{\prime}(u) \nabla u \in L_{M}(\Omega) .
$$

Theorem 2.3. Under assumptions $\left(A_{1}\right)-\left(A_{4}\right),(5)-(8)$, there exists a renormalized solution $u$ (in the sense of definition 2.1) of problem (4).

\section{Proof of the main result}

We state and prove the following lemmas that will be used later

\subsection{Some lemmas}

Lemma 3.1. Assume that $\left(A_{1}\right)-\left(A_{4}\right)$ are satisfied, and let $\left(z_{n}\right)$ be a sequence in $W_{0}^{1} L_{M}(\Omega)$ such that

(i) $z_{n} \rightarrow z$ in $W_{0}^{1} L_{M}(\Omega)$ for $\sigma\left(\Pi L_{M}(\Omega), \Pi E_{\bar{M}}(\Omega)\right)$;

(ii) $\left(a\left(x, z_{n}, \nabla z_{n}\right)\right)_{n}$ is bounded in $\left(L_{\bar{M}}(\Omega)\right)^{N}$;

(iii) $\int_{\Omega}\left[a\left(x, z_{n}, \nabla z_{n}\right)-a\left(x, z_{n}, \nabla z \chi_{s}\right)\right]\left[\nabla z_{n}-\nabla z \chi_{s}\right] d x \rightarrow 0$ as $n, s \rightarrow+\infty$ (where $\chi_{s}$ is the characteristic function of $\left.\Omega_{s}=\{x \in \Omega,|\nabla z| \leq s\}\right)$.

Then

$$
M\left(\frac{\left|\nabla z_{n}\right|}{\lambda}\right) \longrightarrow M\left(\frac{|\nabla z|}{\lambda}\right) \quad \text { in } \quad L^{1}(\Omega)
$$

Remark 3.2. The condition (ii) is not necessary if the $N$-function $M$ satisfies the $\Delta_{2}$-condition. 
Proof of Remark 3.2. The condition (i) implies that the sequence $\left(z_{n}\right)_{n}$ is bounded in $W_{0}^{1} L_{M}(\Omega)$, hence there exists two positive constants $\lambda, C$ such that

$$
\int_{\Omega} M\left(\lambda\left|\nabla z_{n}\right|\right) d x \leq C
$$

On the other hand, let $Q$ be an $N$-function such that $M \ll Q$ and the continuous embedding $W_{0}^{1} L_{M}(\Omega) \subset E_{Q}(\Omega)$ hold (see [11]). Let $\varepsilon>0$. Then there exists $C_{\varepsilon}>0$, as in [7], such that

$$
|a(x, s, \zeta)| \leq c(x)+C_{\varepsilon}+k_{1} \bar{M}^{-1} Q(\varepsilon|s|)+k_{3} \bar{M}^{-1} M(\varepsilon|\zeta|)
$$

for a.e. $x \in \Omega$ and for all $(s, \zeta) \in \mathbb{R} \times \mathbb{R}^{N}$. From (10) and (11) we deduce that $\left(a\left(x, z_{n}, \nabla z_{n}\right)\right)_{n}$ is bounded in $\left(L_{\bar{M}}(\Omega)\right)^{N}$.

Proof of Lemma 3.1. Let $s>0$. Let $\Omega_{s}=\{x \in \Omega,|\nabla u(x)| \leq s\}$ and denote by $\chi_{s}$ the characteristic function of $\Omega_{s}$. Fix $r>0$ and let $s>r$. We have

$$
\begin{aligned}
0 & \leq \int_{\Omega_{r}}\left[a\left(x, z_{n}, \nabla z_{n}\right)-a\left(x, z_{n}, \nabla z\right)\right]\left[\nabla z_{n}-\nabla z\right] d x \\
& \leq \int_{\Omega_{s}}\left[a\left(x, z_{n}, \nabla z_{n}\right)-a\left(x, z_{n}, \nabla z\right)\right]\left[\nabla z_{n}-\nabla z\right] d x \\
& =\int_{\Omega_{s}}\left[a\left(x, z_{n}, \nabla z_{n}\right)-a\left(x, z_{n}, \nabla z \chi_{s}\right)\right]\left[\nabla z_{n}-\nabla z \chi_{s}\right] d x \\
& \leq \int_{\Omega}\left[a\left(x, z_{n}, \nabla z_{n}\right)-a\left(x, z_{n}, \nabla z \chi_{s}\right)\right]\left[\nabla z_{n}-\nabla z \chi_{s}\right] d x,
\end{aligned}
$$

which with (iii) implies

$$
\lim _{n \rightarrow \infty} \int_{\Omega_{r}}\left[a\left(x, z_{n}, \nabla z_{n}\right)-a\left(x, z_{n}, \nabla z\right)\right]\left[\nabla z_{n}-\nabla z\right] d x=0 .
$$

So, as in $[11]$

$$
\nabla z_{n} \longrightarrow \nabla z \quad \text { a.e. in } \Omega \text {. }
$$

On the other hand, we have

$$
\begin{aligned}
\int_{\Omega} a\left(x, z_{n}, \nabla z_{n}\right) \nabla z_{n} d x= & \int_{\Omega}\left[a\left(x, z_{n}, \nabla z_{n}\right)-a\left(x, z_{n}, \nabla z \chi_{s}\right)\right] \\
& \times\left[\nabla z_{n}-\nabla z \chi_{s}\right] d x \\
& +\int_{\Omega} a\left(x, z_{n}, \nabla z \chi_{s}\right)\left(\nabla z_{n}-\nabla z \chi_{s}\right) d x \\
& +\int_{\Omega} a\left(x, z_{n}, \nabla z_{n}\right) \nabla z \chi_{s} d x
\end{aligned}
$$


Since $\left(a\left(x, z_{n}, \nabla z_{n}\right)\right)_{n}$ is bounded in $\left(L_{\bar{M}}(\Omega)\right)^{N}$, and using (12), we obtain

$$
a\left(x, z_{n}, \nabla z_{n}\right) \longrightarrow a(x, z, \nabla z) \text { weakly in }\left(L_{\bar{M}}(\Omega)\right)^{N} \text { for } \sigma\left(\Pi L_{\bar{M}}, \Pi E_{M}\right),
$$

which implies that

$$
\int_{\Omega} a\left(x, z_{n}, \nabla z_{n}\right) \nabla z \chi_{s} d x \longrightarrow \int_{\Omega} a(x, z, \nabla z) \nabla z \chi_{s} d x
$$

as $n \rightarrow \infty$. Letting also $s \rightarrow \infty$, we obtain

$$
\int_{\Omega} a(x, z, \nabla z) \nabla z \chi_{s} d x \longrightarrow \int_{\Omega} a(x, z, \nabla z) \nabla z d x .
$$

On the other hand, it is easy to see that the second term of the right hand side of (13) tends to 0 as $n \rightarrow \infty$. Consequently, from (iii), (14), and (15) we have

$$
\lim _{n \rightarrow \infty} \int_{\Omega} a\left(x, z_{n}, \nabla z_{n}\right) \nabla z_{n} d x=\int_{\Omega} a(x, z, \nabla z) \nabla z d x .
$$

Using $\left(A_{4}\right)$, we obtain, by lemma 1.4 and Vitali's Theorem,

$$
M\left(\frac{\left|\nabla z_{n}\right|}{\lambda}\right) \rightarrow M\left(\frac{|\nabla z|}{\lambda}\right) \text { in } L^{1}(\Omega) .
$$

The following lemma will be used in the proof of the propositions 3.4 and 3.5.

Lemma 3.3. Let $\Omega$ be an open bounded subset of $\mathbb{R}^{N}$ satisfying the segment property. If $u \in W_{0}^{1} L_{M}(\Omega)$, then

$$
\int_{\Omega} \operatorname{div} u d x=0
$$

For the proof we refer to [4].

\subsection{The approximate problem}

Let us define, for each $k>0$, the truncation

$$
T_{k}(s)=\left\{\begin{array}{lll}
s & \text { if } & |s| \leq k \\
k \frac{s}{|s|} & \text { if } & |s|>k
\end{array}\right.
$$

and, for each $n \in \mathbb{N}^{*}$, the approximations

$$
\phi_{n}(s)=\phi\left(T_{n}(s)\right), \quad g_{n}(x, t)=T_{n}(g(x, t)) .
$$

Consider the nonlinear elliptic problem

$$
\left\{\begin{array}{l}
u_{n} \in W_{0}^{1} L_{M}(\Omega) \\
-\operatorname{div} a\left(x, u_{n}, \nabla u_{n}\right)-\operatorname{div} \phi_{n}\left(u_{n}\right)+g_{n}\left(x, u_{n}\right)=f \text { in } \mathcal{D}^{\prime}(\Omega),
\end{array}\right.
$$


which is equivalent to

$$
\left\{\begin{array}{l}
u_{n} \in W_{0}^{1} L_{M}(\Omega) \\
-\operatorname{div} \tilde{a}\left(x, u_{n}, \nabla u_{n}\right)+g_{n}\left(x, u_{n}\right)=f \quad \text { in } \quad \mathcal{D}^{\prime}(\Omega)
\end{array}\right.
$$

where $\tilde{a}(x, t, \xi)=a(x, t, \xi)+\phi_{n}(t)$.

Since $\left|T_{n}(t)\right| \leq n$ and $\phi$ is continuous, we have $\left|\phi_{n}(t)\right|=\left|\phi\left(T_{n}(t)\right)\right| \leq c_{n}$. From Gossez and Mustonen [13, Proposition 1 and Remark 2], the problem (16), and its equivalent (17), have at least one solution $u_{n}$.

\subsection{Some intermediate results}

Proposition 3.4. Assume that $\left(A_{1}\right)-\left(A_{4}\right),(5)-(8)$ hold true, and let $u_{n}$ be a solution of the approximate problem (16). Then we have the following properties:

(i) $\left(u_{n}\right)_{n}$ is bounded in $W_{0}^{1} L_{M}(\Omega)$, and there exists a function $u$ in $W_{0}^{1} L_{M}(\Omega)$ such that

$$
\begin{array}{ll}
u_{n} \longrightarrow u & \text { weakly in } W_{0}^{1} L_{M}(\Omega) \text { for } \sigma\left(\Pi L_{M}, \Pi E_{\bar{M}}\right), \\
u_{n} \longrightarrow u & \text { strongly in } E_{M}(\Omega) \text { and a.e. in } \Omega .
\end{array}
$$

(ii) $\left(a\left(x, u_{n}, \nabla u_{n}\right)\right)_{n}$ is bounded in $L_{\bar{M}}(\Omega)$.

(iii) $g_{n}\left(x, u_{n}\right) \rightarrow g(x, u)$ strongly in $L^{1}(\Omega)$.

Proof. We divide the proof in several steps.

Step 1: Boundedness of $\left(u_{n}\right)_{n}$ in $W_{0}^{1} L_{M}(\Omega)$. Taking $u_{n}$ as test function in (16), we obtain

$$
\int_{\Omega} a\left(x, u_{n}, \nabla u_{n}\right) \nabla u_{n} d x+\int_{\Omega} \phi\left(T_{n}\left(u_{n}\right)\right) \nabla u_{n} d x+\int_{\Omega} g_{n}\left(x, u_{n}\right) u_{n} d x \leq\left\langle f, u_{n}\right\rangle .
$$

Define $\tilde{\phi_{n}}(t)=\int_{0}^{t} \phi_{n}(\tau) d \tau$. We have $\tilde{\phi_{n}}\left(u_{n}\right) \in\left(W_{0}^{1} L_{M}(\Omega)\right)^{N}$. (We can apply Lemma 1.1 since each component of $\tilde{\phi}_{n}$ is uniformly Lipschitzian and $\tilde{\phi}_{n}(0)=0$.) We obtain

$$
\int_{\Omega} \phi_{n}\left(u_{n}\right) \nabla u_{n} d x=\int_{\Omega} \operatorname{div}\left(\tilde{\phi}_{n}\left(u_{n}\right)\right) d x=0 .
$$

(See Lemma 3.3.) By (7), we get

$$
\begin{aligned}
\int_{\Omega} a\left(x, u_{n}, \nabla u_{n}\right) \nabla u_{n} d x & \leq\left\langle f, u_{n}\right\rangle . \\
\int_{\Omega} g_{n}\left(x, u_{n}\right) u_{n} d x & \leq\left\langle f, u_{n}\right\rangle .
\end{aligned}
$$


On the other hand, $f$ can be written as $f=f_{0}-\operatorname{div} F$ where $f_{0} \in E_{\bar{M}}(\Omega)$, $F \in\left(E_{\bar{M}}(\Omega)\right)^{N}$. Using [11, Lemma 5.7] and Young's inequality we deduce

$$
\begin{gathered}
\int_{\Omega} f_{0} u_{n} d x \leq C_{1}+\frac{\alpha}{4} \int_{\Omega} M\left(\left|\nabla u_{n}\right|\right) d x, \\
\int_{\Omega} F \nabla u_{n} d x \leq C_{2}+\frac{\alpha}{4} \int_{\Omega} M\left(\left|\nabla u_{n}\right|\right) d x .
\end{gathered}
$$

Combining (18) and (20), we get

$$
\int_{\Omega} a\left(x, u_{n}, \nabla u_{n}\right) \nabla u_{n} d x \leq \frac{\alpha}{2} \int_{\Omega} M\left(\left|\nabla u_{n}\right|\right) d x+C_{3} .
$$

This implies, by using $\left(A_{4}\right)$, that

$$
\int_{\Omega} M\left(\left|\nabla u_{n}\right|\right) d x \leq C_{4}
$$

which gives

$$
u_{n} \longrightarrow u \quad \text { weakly in } W_{0}^{1} L_{M}(\Omega) \text { for } \sigma\left(\Pi L_{M}, \Pi E_{\bar{M}}\right) \text {. }
$$

Using the compact embedding $W_{0}^{1} L_{M}(\Omega) \hookrightarrow E_{M}(\Omega)$, we get

$$
u_{n} \longrightarrow u \text { strongly in } E_{M}(\Omega) \text { and a.e. in } \Omega \text {. }
$$

Step 2: Boundedness of $\left(a\left(x, u_{n}, \nabla u_{n}\right)\right)_{n}$ in $\left(L_{\bar{M}}(\Omega)\right)^{N}$. Let $w \in\left(E_{M}(\Omega)\right)^{N}$ be arbitrary. By $\left(A_{3}\right)$, we have

$$
\left(a\left(x, u_{n}, \nabla u_{n}\right)-a\left(x, u_{n}, w\right)\right)\left(\nabla u_{n}-w\right)>0,
$$

which implies that

$$
\begin{array}{rl}
\int_{\Omega} a\left(x, u_{n}, \nabla u_{n}\right) w & d x \\
\leq \int_{\Omega} a\left(x, u_{n}, \nabla u_{n}\right) \nabla u_{n} d x+\int_{\Omega} a\left(x, u_{n}, w\right)\left(w-\nabla u_{n}\right) d x .
\end{array}
$$

Combining (21) and (22), we get

$$
\int_{\Omega} a\left(x, u_{n}, \nabla u_{n}\right) \nabla u_{n} d x \leq C_{5},
$$

with $C_{5}$ a positive constant.

On the other hand, for $\lambda$ large and using $\left(A_{2}\right)$, we have

$$
\int_{\Omega} \bar{M}\left(\frac{a\left(x, u_{n}, w\right)}{\lambda}\right) d x \leq \int_{\Omega} \bar{M}\left(\frac{c(x)}{\lambda}\right)+\int_{\Omega} \frac{k_{3}}{\lambda} M\left(k_{4}|w|\right)+C_{7} \leq C_{8} .
$$


Thus, $\left|a\left(x, u_{n}, w\right)\right|$ is bounded in $L_{\bar{M}}(\Omega)$. This condition, additionally to (22), implies that the second term of the right hand side of (23) is bounded. Consequently, we obtain

$$
\int_{\Omega} a\left(x, u_{n}, \nabla u_{n}\right) w d x \leq C_{9},
$$

with $C_{9}$ a positive constant. Hence, thanks to the Banach-Steinhaus theorem, the sequence $\left(a\left(x, u_{n}, \nabla u_{n}\right)\right)_{n}$ is bounded in $\left(L_{\bar{M}}(\Omega)\right)^{N}$.

Step 3: Strongly convergence of the nonlinearity. Since $g_{n}\left(x, u_{n}\right) \rightarrow g(x, u)$ a.e. in $\Omega$, by the sign condition (7) and Fatou's Lemma we obtain from (19) and (22) that

$$
g(x, u) u \in L^{1}(\Omega),
$$

and by Vitali's theorem we have

$$
g_{n}\left(x, u_{n}\right) \longrightarrow g(x, u) \text { strongly in } L^{1}(\Omega),
$$

which completes the proof.

Proposition 3.5. Assume that $\left(A_{1}\right)-\left(A_{4}\right),(5)-(8)$ hold true, and let $u_{n}$ be a solution of the approximate problem (16). Then, we have (for a subsequence noted again $u_{n}$ )

$$
\nabla u_{n} \longrightarrow \nabla u \quad \text { a.e. in } \Omega \text {. }
$$

Proof. Again we divide the proof in several steps.

Step 1. $\limsup _{n \rightarrow+\infty} \int_{\left\{\left|u_{n}\right|>h\right\}} a\left(x, u_{n}, \nabla u_{n}\right) \nabla u_{n} d x \leq\left\langle f, u-T_{h}(u)\right\rangle$ where $h>0$.

The idea is to use in (16) the test function $u_{n}-T_{h}\left(u_{n}\right)$ (which is in $W_{0}^{1} L_{M}(\Omega)$ ).

Consider

$$
\begin{aligned}
& \theta(t)=\phi_{n}(t) \chi_{\{s \in \mathbb{R},|s| \geq h\}}(t), \\
& \tilde{\theta}(t)=\int_{0}^{t} \theta(\tau) d \tau,
\end{aligned}
$$

hence $\tilde{\theta}\left(u_{n}\right) \in\left(W_{0}^{1} L_{M}(\Omega)\right)^{N}$ (by Lemma 1.1). We obtain, by Lemma 3.3,

$$
\begin{aligned}
\int_{\Omega} \phi_{n}\left(u_{n}\right) \nabla\left(u_{n}-T_{h}\left(u_{n}\right)\right) d x & =\int_{\Omega} \phi_{n}\left(u_{n}\right) \chi_{\{s \in \mathbb{R},|s| \geq h\}}\left(u_{n}\right) \nabla u_{n} d x \\
& =\int_{\Omega} \theta\left(u_{n}\right) \nabla u_{n} d x=\int_{\Omega} \operatorname{div}\left(\tilde{\theta}\left(u_{n}\right)\right) d x=0 .
\end{aligned}
$$

Using the sign condition $(7)$ we have $g_{n}\left(x, u_{n}\right)\left(u_{n}-T_{h}\left(u_{n}\right)\right) \geq 0$ a.e. in $\Omega$. Then, for any fixed $h>0$, we have

$$
\int_{\left\{\left|u_{n}\right|>h\right\}} a\left(x, u_{n}, \nabla u_{n}\right) \nabla u_{n} d x \leq\left\langle f, u_{n}-T_{h}\left(u_{n}\right)\right\rangle .
$$


Since $u_{n}-T_{h}\left(u_{n}\right) \rightarrow u-T_{h}(u)$ weakly in $W_{0}^{1} L_{M}(\Omega)$ and $f \in W^{-1} E_{\bar{M}}(\Omega)$, we have

$$
\limsup _{n \rightarrow+\infty} \int_{\left\{\left|u_{n}\right|>h\right\}} a\left(x, u_{n}, \nabla u_{n}\right) \nabla u_{n} d x \leq\left\langle f, u-T_{h}(u)\right\rangle .
$$

Step 2. We shall prove that $\nabla u_{n} \rightarrow \nabla u$ a.e. in $\Omega$.

By Lemma 1.5 there exists a sequence $v_{j} \in \mathcal{D}(\Omega)$ which converges to $u$ for the modular convergence in $W_{0}^{1} L_{M}(\Omega)$. Let $s, j>0$. Let $\Omega_{s}^{j}=\left\{x \in \Omega,\left|\nabla v_{j}(x)\right| \leq s\right\}$ and denote by $\chi_{s}^{j}$ the characteristic function of $\Omega_{s}^{j}$. We will note by $\epsilon(n, j, h)$ any quantity such that

$$
\lim _{h \rightarrow+\infty} \lim _{j \rightarrow+\infty} \lim _{n \rightarrow+\infty} \epsilon(n, j, h)=0 .
$$

If the quantity we consider does not depend on one parameter among $n, j$, and $h$, we will omit the dependence on the corresponding parameter: as an example, $\epsilon(n, h)$ is any quantity such that

$$
\lim _{h \rightarrow+\infty} \lim _{n \rightarrow+\infty} \epsilon(n, h)=0 .
$$

Finally, we will note (for example) by $\epsilon_{h}(n, j)$ a quantity that depends on $n, j, h$, and is such that

$$
\lim _{j \rightarrow+\infty} \lim _{n \rightarrow+\infty} \epsilon_{h}(n, j)=0
$$

for any fixed value of $h$.

We have

$$
\begin{aligned}
\int_{\Omega}\left[a\left(x, u_{n}, \nabla u_{n}\right)-a(x,\right. & \left.\left.u_{n}, \nabla u \chi_{s}\right)\right]\left[\nabla u_{n}-\nabla u \chi_{s}\right] d x \\
= & \int_{\Omega}\left[a\left(x, u_{n}, \nabla u_{n}\right)-a\left(x, u_{n}, \nabla v_{j} \chi_{s}^{j}\right)\right]\left[\nabla u_{n}-\nabla v_{j} \chi_{s}^{j}\right] d x \\
& +\int_{\Omega} a\left(x, u_{n}, \nabla v_{j} \chi_{s}^{j}\right)\left(\nabla u_{n}-\nabla v_{j} \chi_{s}^{j}\right) d x \\
& -\int_{\Omega} a\left(x, u_{n}, \nabla u \chi_{s}\right)\left(\nabla u_{n}-\nabla u \chi_{s}\right) d x \\
& +\int_{\Omega} a\left(x, u_{n}, \nabla u_{n}\right)\left(\nabla v_{j} \chi_{s}^{j}-\nabla u \chi_{s}\right) d x
\end{aligned}
$$

We pass to the limit in $n$ and $j$ in the last three terms of the right hand side of the last equality, we get

$$
\begin{array}{r}
\int_{\Omega} a\left(x, u_{n}, \nabla v_{j} \chi_{s}^{j}\right)\left[\nabla u_{n}-\nabla v_{j} \chi_{s}^{j}\right] d x-\int_{\Omega} a\left(x, u_{n}, \nabla u \chi_{s}\right)\left[\nabla u_{n}-\nabla u \chi_{s}\right] d x \\
=\epsilon(n, j),
\end{array}
$$

and

$$
\int_{\Omega} a\left(x, u_{n}, \nabla u_{n}\right)\left[\nabla v_{j} \chi_{s}^{j}-\nabla u \chi_{s}\right] d x=\epsilon(n, j) .
$$


This implies

$$
\begin{aligned}
\int_{\Omega}\left[a\left(x, u_{n}, \nabla u_{n}\right)-a\left(x, u_{n}, \nabla u \chi_{s}\right)\right]\left[\nabla u_{n}-\nabla u \chi_{s}\right] d x & \\
& =\int_{\Omega}\left[a\left(x, u_{n}, \nabla u_{n}\right)-a\left(x, u_{n}, \nabla v_{j} \chi_{s}^{j}\right)\right]\left[\nabla u_{n}-\nabla v_{j} \chi_{s}^{j}\right] d x+\epsilon(n, j) .
\end{aligned}
$$

The term in the right hand side of the last equality can be estimated as follows:

$$
\begin{aligned}
& \int_{\Omega}\left[a\left(x, u_{n}, \nabla u_{n}\right)-a\left(x, u_{n}, \nabla v_{j} \chi_{s}^{j}\right)\right]\left[\nabla u_{n}-\nabla v_{j} \chi_{s}^{j}\right] d x \\
& \leq \int_{\left\{\left|u_{n}-T_{h}\left(v_{j}\right)\right| \leq 2 h\right\}}\left[a\left(x, u_{n}, \nabla u_{n}\right)-a\left(x, u_{n}, \nabla v_{j} \chi_{s}^{j}\right)\right]\left[\nabla u_{n}-\nabla v_{j} \chi_{s}^{j}\right] d x \\
& \quad+\int_{\left\{\left|u_{n}\right|>h\right\}}\left[a\left(x, u_{n}, \nabla u_{n}\right)-a\left(x, u_{n}, \nabla v_{j} \chi_{s}^{j}\right)\right]\left[\nabla u_{n}-\nabla v_{j} \chi_{s}^{j}\right] d x .
\end{aligned}
$$

The first term of the right hand side of (26) can be written as

$$
\begin{aligned}
& \int_{\left\{\left|u_{n}-T_{h}\left(v_{j}\right)\right| \leq 2 h\right\}}\left[a\left(x, u_{n}, \nabla u_{n}\right)-a\left(x, u_{n}, \nabla v_{j} \chi_{s}^{j}\right)\right]\left[\nabla u_{n}-\nabla v_{j} \chi_{s}^{j}\right] d x \\
&=\int_{\left\{\left|u_{n}-T_{h}\left(v_{j}\right)\right| \leq 2 h\right\}} a\left(x, u_{n}, \nabla u_{n}\right) \nabla\left(u_{n}-T_{h}\left(v_{j}\right)\right) d x \\
&+\int_{\left\{\left|u_{n}-T_{h}\left(v_{j}\right)\right| \leq 2 h\right\}} a\left(x, u_{n}, \nabla u_{n}\right)\left(\nabla T_{h}\left(v_{j}\right)-\nabla v_{j} \chi_{s}^{j}\right) d x \\
&-\int_{\left\{\left|u_{n}-T_{h}\left(v_{j}\right)\right| \leq 2 h\right\}} a\left(x, u_{n}, \nabla v_{j} \chi_{s}^{j}\right)\left[\nabla u_{n}-\nabla v_{j} \chi_{s}^{j}\right] d x
\end{aligned}
$$

If we take $T_{2 h}\left(u_{n}-T_{h}\left(v_{j}\right)\right)$ as test function in (16), we have for $n$ large enough

$$
\begin{aligned}
\int_{\Omega} a\left(x, u_{n}, \nabla u_{n}\right) & \nabla T_{2 h}\left(u_{n}-T_{h}\left(v_{j}\right)\right) d x+\int_{\Omega} \phi\left(u_{n}\right) \nabla T_{2 h}\left(u_{n}-T_{h}\left(v_{j}\right)\right) d x \\
& +\int_{\Omega} g_{n}\left(x, u_{n}\right) T_{2 h}\left(u_{n}-T_{h}\left(v_{j}\right)\right) d x=\left\langle f, T_{2 h}\left(u_{n}-T_{h}\left(v_{j}\right)\right)\right\rangle .
\end{aligned}
$$

Using (i) of proposition 3.4 and the modular convergence of $v_{j}$, we have

$$
\begin{gathered}
\int_{\Omega} \phi\left(u_{n}\right) \nabla T_{2 h}\left(u_{n}-T_{h}\left(v_{j}\right)\right) d x=\int_{\Omega} \phi(u) \nabla T_{2 h}\left(u-T_{h}(u)\right) d x+\epsilon_{h}(n, j)=\epsilon(n, j, h), \\
\int_{\Omega} g_{n}\left(x, u_{n}\right) T_{2 h}\left(u_{n}-T_{h}\left(v_{j}\right)\right) d x=\epsilon(n, j, h), \\
\left\langle f, T_{2 h}\left(u_{n}-T_{h}\left(v_{j}\right)\right)\right\rangle=\epsilon(n, j, h)
\end{gathered}
$$


which, with (28), implies that

$$
\int_{\Omega} a\left(x, u_{n}, \nabla u_{n}\right) \nabla T_{2 h}\left(u_{n}-T_{h}\left(v_{j}\right)\right) d x=\epsilon(n, j, h) .
$$

Now, since $\left(a\left(x, u_{n}, \nabla u_{n}\right)\right)_{n}$ is bounded in $\left(L_{\bar{M}}(\Omega)\right)^{N}$, we have, for a subsequence

$$
a\left(x, u_{n}, \nabla u_{n}\right) \rightarrow \rho
$$

weakly in $\left(L_{\bar{M}}(\Omega)\right)^{N}$ for $\left(\sigma\left(L_{\bar{M}}(\Omega), E_{M}(\Omega)\right)\right)^{N}$ as $n$ tends to infinity, that

$$
\begin{aligned}
\int_{\left\{\left|u_{n}-T_{h}\left(v_{j}\right)\right| \leq 2 h\right\}} a\left(x, u_{n}, \nabla u_{n}\right)\left(\nabla T_{h}\left(v_{j}\right)-\nabla v_{j} \chi_{s}^{j}\right) d x & \\
& \longrightarrow \int_{\left\{\left|u-T_{h}\left(v_{j}\right)\right| \leq 2 h\right\}} \rho\left(\nabla T_{h}\left(v_{j}\right)-\nabla v_{j} \chi_{s}^{j}\right) d x
\end{aligned}
$$

as $n$ tends to infinity.

Using now the modular convergence of $v_{j}$, we get

$$
\begin{aligned}
\int_{\left\{\left|u-T_{h}\left(v_{j}\right)\right| \leq 2 h\right\}} \rho\left(\nabla T_{h}\left(v_{j}\right)-\nabla v_{j} \chi_{s}^{j}\right) d x & \\
& \longrightarrow \int_{\left\{\left|u-T_{h}(u)\right| \leq 2 h\right\}} \rho\left(\nabla T_{h}(u)-\nabla u \chi_{s}\right) d x
\end{aligned}
$$

as $j$ tends to infinity. Letting also $h$ to infinity, we can easy deduce

$$
\int_{\left\{\left|u-T_{h}(u)\right| \leq 2 h\right\}} \rho\left(\nabla T_{h}(u)-\nabla u \chi_{s}\right) d x \longrightarrow \int_{\Omega \backslash \Omega_{s}} \rho \nabla u d x .
$$

Finally

$$
\begin{aligned}
\int_{\left\{\left|u_{n}-T_{h}\left(v_{j}\right)\right| \leq 2 h\right\}} a\left(x, u_{n}, \nabla u_{n}\right)\left(\nabla T_{h}\left(v_{j}\right)-\nabla v_{j} \chi_{s}^{j}\right) d x & \\
= & \int_{\Omega \backslash \Omega_{s}} \rho \nabla u d x+\epsilon(n, j, h) .
\end{aligned}
$$

For the third term of the right hand side of (27), we have for a subsequence (use Lemma 1.3)

$$
\begin{aligned}
a\left(x, u_{n}, \nabla v_{j} \chi_{s}^{j}\right) \chi_{\left\{\left|u_{n}-T_{h}\left(v_{j}\right)\right| \leq 2 h\right\}} \longrightarrow a\left(x, u, \nabla v_{j} \chi_{s}^{j}\right) \chi_{\left\{\left|u-T_{h}\left(v_{j}\right)\right| \leq 2 h\right\}} \\
\text { strongly in }\left(L_{\bar{M}}(\Omega)\right)^{N} \quad \text { for } \sigma\left(L_{\bar{M}}(\Omega), E_{M}(\Omega)\right)
\end{aligned}
$$

as $n$ tends to infinity, and

$$
u_{n} \longrightarrow u \quad \text { weakly in } W_{0}^{1} L_{M}(\Omega) \text { for } \sigma\left(\Pi L_{\bar{M}}(\Omega), \Pi E_{M}(\Omega)\right)
$$


as $n$ tends to infinity. Hence

$$
\begin{aligned}
& \int_{\left\{\left|u_{n}-T_{h}\left(v_{j}\right)\right| \leq 2 h\right\}} a\left(x, u_{n}, \nabla v_{j} \chi_{s}^{j}\right)\left[\nabla u_{n}-\nabla v_{j} \chi_{s}^{j}\right] d x \\
& \longrightarrow \int_{\left\{\left|u-T_{h}\left(v_{j}\right)\right| \leq 2 h\right\}} a\left(x, u, \nabla v_{j} \chi_{s}^{j}\right)\left[\nabla u-\nabla v_{j} \chi_{s}^{j}\right] d x .
\end{aligned}
$$

Using now the modular convergence of $\left(v_{j}\right)$, we get

$$
\begin{aligned}
& \int_{\left\{\left|u-T_{h}\left(v_{j}\right)\right| \leq 2 h\right\}} a\left(x, u, \nabla v_{j} \chi_{s}^{j}\right)\left[\nabla u-\nabla v_{j} \chi_{s}^{j}\right] d x \\
& \longrightarrow \int_{\left\{\left|u-T_{h}(u)\right| \leq 2 h\right\}} a\left(x, u, \nabla u \chi_{s}\right)\left[\nabla u-\nabla u \chi_{s}\right] d x=0
\end{aligned}
$$

as $j$ tends to infinity.

Finally,

$$
\int_{\left\{\left|u_{n}-T_{h}\left(v_{j}\right)\right| \leq 2 h\right\}} a\left(x, u_{n}, \nabla v_{j} \chi_{s}^{j}\right)\left[\nabla u_{n}-\nabla v_{j} \chi_{s}^{j}\right] d x=\epsilon(n, j, h) .
$$

Combining (27), (29), (31), and (32), we deduce

$$
\begin{aligned}
\int_{\left\{\left|u_{n}-T_{h}\left(v_{j}\right)\right| \leq 2 h\right\}}\left[a\left(x, u_{n}, \nabla u_{n}\right)-a\left(x, u_{n}, \nabla v_{j} \chi_{s}^{j}\right)\right][ & \left.\nabla u_{n}-\nabla v_{j} \chi_{s}^{j}\right] d x \\
= & \int_{\Omega \backslash \Omega_{s}} \rho \nabla u d x+\epsilon(n, j, h) .
\end{aligned}
$$

The second term of the right hand side of the (26) can be written as

$$
\begin{aligned}
& \int_{\left\{\left|u_{n}\right|>h\right\}}\left[a\left(x, u_{n}, \nabla u_{n}\right)-a\left(x, u_{n}, \nabla v_{j} \chi_{s}^{j}\right)\right]\left[\nabla u_{n}-\nabla v_{j} \chi_{s}^{j}\right] d x \\
&=\int_{\left\{\left|u_{n}\right|>h\right\}} a\left(x, u_{n}, \nabla u_{n}\right) \nabla u_{n} d x \\
&-\int_{\left\{\left|u_{n}\right|>h\right\}} a\left(x, u_{n}, \nabla u_{n}\right) \nabla v_{j} \chi_{s}^{j} d x \\
&\left.-\int_{\left\{\left|u_{n}\right|>h\right\}} a\left(x, u_{n}, \nabla v_{j} \chi_{s}^{j}\right)\right]\left[\nabla u_{n}-\nabla v_{j} \chi_{s}^{j}\right] d x .
\end{aligned}
$$

Letting $h$ to infinity in (24), we get

$$
\int_{\left\{\left|u_{n}\right|>h\right\}} a\left(x, u_{n}, \nabla u_{n}\right) \nabla u_{n} d x \leq \epsilon(n, h),
$$


and, reasoning as above, it is easy to see that

$$
\begin{array}{r}
\int_{\left\{\left|u_{n}\right|>h\right\}} a\left(x, u_{n}, \nabla u_{n}\right) \nabla v_{j} \chi_{s}^{j} d x=\epsilon(n, j, h), \\
\left.\int_{\left\{\left|u_{n}\right|>h\right\}} a\left(x, u_{n}, \nabla v_{j} \chi_{s}^{j}\right)\right]\left[\nabla u_{n}-\nabla v_{j} \chi_{s}^{j}\right] d x=\epsilon(n, j, h) .
\end{array}
$$

Finally

$$
\int_{\left\{\left|u_{n}\right|>h\right\}}\left[a\left(x, u_{n}, \nabla u_{n}\right)-a\left(x, u_{n}, \nabla v_{j} \chi_{s}^{j}\right)\right]\left[\nabla u_{n}-\nabla v_{j} \chi_{s}^{j}\right] d x=\epsilon(n, j, h) .
$$

Combining (33) and (34), we deduce from (26) that

$$
\begin{aligned}
& \int_{\Omega}\left[a\left(x, u_{n}, \nabla u_{n}\right)-a\left(x, u_{n}, \nabla v_{j} \chi_{s}^{j}\right)\right]\left[\nabla u_{n}-\nabla v_{j} \chi_{s}^{j}\right] d x \\
& \leq \int_{\Omega \backslash \Omega_{s}} \rho \nabla u d x+\epsilon(n, j, h) .
\end{aligned}
$$

Letting $s$ to infinity, we get by using (25) and (35)

$$
\int_{\Omega}\left[a\left(x, u_{n}, \nabla u_{n}\right)-a\left(x, u_{n}, \nabla u \chi_{s}\right)\right]\left[\nabla u_{n}-\nabla u \chi_{s}\right] d x \longrightarrow 0
$$

as $n, s \rightarrow \infty$. Using Lemma 3.1 we can conclude the result of Proposition 3.5.

Proof of Theorem 2.3. Step 1. We shall prove that

$$
a\left(x, u_{n}, \nabla u_{n}\right) \nabla u_{n} \longrightarrow a(x, u, \nabla u) \nabla u \text { strongly in } L^{1}(\Omega) .
$$

We have

$$
\begin{aligned}
\int_{\Omega} a\left(x, u_{n}, \nabla u_{n}\right) \nabla u_{n} d x= & \int_{\Omega}\left[a\left(x, u_{n}, \nabla u_{n}\right)-a\left(x, u_{n}, \nabla u \chi_{s}\right)\right]\left[\nabla u_{n}-\nabla u \chi_{s}\right] d x \\
& +\int_{\Omega} a\left(x, u_{n}, \nabla u_{n}\right) \nabla u \chi_{s} d x \\
& +\int_{\Omega} a\left(x, u_{n}, \nabla u \chi_{s}\right)\left[\nabla u_{n}-\nabla u \chi_{s}\right] d x .
\end{aligned}
$$

By (36) the first term of the last equality tends to 0. By the Proposition 3.5 and (30), we have

$$
\int_{\Omega} a\left(x, u_{n}, \nabla u_{n}\right) \nabla u \chi_{s} d x \longrightarrow \int_{\Omega} a(x, u, \nabla u) \nabla u \chi_{s} d x
$$

as $n$ tends to infinity. Letting also $s$ to infinity, we get

$$
\int_{\Omega} a\left(x, u_{n}, \nabla u_{n}\right) \nabla u \chi_{s} d x \longrightarrow \int_{\Omega} a(x, u, \nabla u) \nabla u d x
$$


The third term of the last equality tends to 0 as $n$ and $s \rightarrow \infty$. We deduce

$$
\int_{\Omega} a\left(x, u_{n}, \nabla u_{n}\right) \nabla u_{n} d x \longrightarrow \int_{\Omega} a(x, u, \nabla u) \nabla u d x .
$$

Using Lemma 1.4 we get the result.

Step 2. Passing to the limit. Using in (16) the test function $h\left(u_{n}\right) \varphi$ with $h \in C_{c}^{1}(\mathbb{R})$ and $\varphi \in \mathcal{D}(\Omega)$, we obtain

$$
\begin{aligned}
\int_{\Omega} a\left(x, u_{n}, \nabla u_{n}\right) \nabla u_{n} h^{\prime}\left(u_{n}\right) \varphi d x+\int_{\Omega} a\left(x, u_{n}, \nabla u_{n}\right) \nabla \varphi h\left(u_{n}\right) d x \\
+\int_{\Omega} \phi_{n}\left(u_{n}\right) \nabla\left(h\left(u_{n}\right) \varphi\right) d x+\int_{\Omega} g_{n}\left(x, u_{n}\right) h\left(u_{n}\right) \varphi d x=\left\langle f, h\left(u_{n}\right) \varphi\right\rangle .
\end{aligned}
$$

We shall pass to the limit in each term of last equality.

Since $h$ and $h^{\prime}$ have compact support on $\mathbb{R}$, there exist $\eta>0$ such that supp $h$ and $\operatorname{supp} h^{\prime} \in[-\eta, \eta]$. We have for $n$ large enough

$$
\begin{aligned}
\phi_{n}(t) h(t) & =\phi\left(T_{n}(t)\right) h(t)=\phi\left(T_{\eta}(t)\right) h(t), \\
\phi_{n}(t) h^{\prime}(t) & =\phi\left(T_{n}(t)\right) h^{\prime}(t)=\phi\left(T_{\eta}(t)\right) h^{\prime}(t)
\end{aligned}
$$

and the functions $\phi h$ and $\phi h^{\prime}$ belong to $\left(C^{0}(\mathbb{R}) \cap L^{\infty}(\mathbb{R})\right)^{N}$.

First we have that $h\left(u_{n}\right) \varphi$ is bounded in $W_{0}^{1} L_{M}(\Omega)$. Indeed, since $u_{n}$ is bounded in $W_{0}^{1} L_{M}(\Omega)$ there exists two constants positive $c, \lambda>0$ such that $\int_{\Omega} M\left(\frac{\left|\nabla u_{n}\right|}{\lambda}\right) d x \leq c$. Let $c_{1}$ be a constant positive such that $\left\|h\left(u_{n}\right)|\nabla \varphi|\right\|_{\infty} \leq c_{1}$ and $\left\|h^{\prime}\left(u_{n}\right) \varphi\right\|_{\infty} \leq c_{1}$. For $\mu$ large enough, we have

$$
\begin{aligned}
& \int_{\Omega} M\left(\frac{h\left(u_{n}\right) \nabla \varphi+h^{\prime}\left(u_{n}\right) \varphi\left|\nabla u_{n}\right|}{\mu}\right) d x \\
& \leq \int_{\Omega} M\left(\frac{c_{1}+c_{2}\left|\nabla u_{n}\right| / \lambda}{\mu}\right) d x \quad \text { with } \quad c_{2}=c_{1} \lambda \\
& \leq c_{3}+\frac{c_{2}}{\mu} \int_{\Omega} M\left(\frac{\left|\nabla u_{n}\right|}{\lambda}\right) d x \leq c_{4} \text {. }
\end{aligned}
$$

This implies that

$$
h\left(u_{n}\right) \varphi \longrightarrow h(u) \varphi \quad \text { weakly in } W_{0}^{1} L_{M}(\Omega) \text { for } \sigma\left(\prod L_{M}, \prod E_{\bar{M}}\right) .
$$

By the convergence of (40), and since

$$
\phi\left(T_{\eta}\left(u_{n}\right)\right) \longrightarrow \phi\left(T_{\eta}(u)\right) \text { strongly in }\left(E_{\bar{M}}\right)^{N},
$$

the third term of $(39)$ tends to $\int_{\Omega} \phi\left(T_{\eta}(u)\right) \nabla(h(u) \varphi) d x$, and the right hand side of (39) tends to $\langle f, h(u) \varphi\rangle$. For the first term of (39), we remark that

$$
\left|a\left(x, u_{n}, \nabla u_{n}\right) \nabla u_{n} h^{\prime}\left(u_{n}\right) \varphi\right| \leq c_{1} a\left(x, u_{n}, \nabla u_{n}\right) \nabla u_{n}
$$


consequently, Vitali's theorem and (37) give that

$$
\int_{\Omega} a\left(x, u_{n}, \nabla u_{n}\right) \nabla u_{n} h^{\prime}\left(u_{n}\right) \varphi d x \longrightarrow \int_{\Omega} a(x, u, \nabla u) \nabla u h^{\prime}(u) \varphi d x .
$$

For the second term of (39), we have

$$
h\left(u_{n}\right) \nabla \varphi \longrightarrow h(u) \nabla \varphi \quad \text { strongly in }\left(E_{M}(\Omega)\right)^{N},
$$

and

$$
a\left(x, u_{n}, \nabla u_{n}\right) \rightarrow a(x, u, \nabla u) \quad \text { weakly in }\left(L_{\bar{M}}(\Omega)\right)^{N} \quad \text { for } \sigma\left(\prod L_{\bar{M}}, \prod E_{M}\right),
$$

then

$$
\int_{\Omega} a\left(x, u_{n}, \nabla u_{n}\right) \nabla \varphi h\left(u_{n}\right) d x \longrightarrow \int_{\Omega} a(x, u, \nabla u) \nabla \varphi h(u) d x .
$$

The fourth term of (39) tends to $\int_{\Omega} g(x, u) h(u) \varphi d x$.

Using the limite proved above we can easily pass to the limit in each term of (39) and obtain

$$
\begin{aligned}
\int_{\Omega} a(x, u, \nabla u)\left[h^{\prime}(u) \varphi \nabla u+h(u) \nabla \varphi\right] d x & \\
+\int_{\Omega} \phi(u) h^{\prime}(u) \varphi \nabla u d x+\int_{\Omega} \phi(u) & h(u) \nabla \varphi d x+\int_{\Omega} g(x, u) h(u) \varphi d x \\
& =\langle f, h(u) \varphi\rangle \quad \forall h \in \mathcal{C}_{c}^{1}(\mathbb{R}), \forall \varphi \in \mathcal{D}(\Omega),
\end{aligned}
$$

which proves Theorem 2.3.

\section{References}

[1] R. A. Adams, Sobolev spaces, Pure and Applied Mathematics, vol. 65, Academic Press, New York-London, 1975.

[2] L. Aharouch, A. Benkirane, and M. Rhoudaf, Strongly nonlinear elliptic variational unilateral problems in Orlicz spaces, Abstr. Appl. Anal. (2006), 1-20.

[3] L. Aharouch and M. Rhoudaf, Strongly nonlinear elliptic unilateral problems in Orlicz space and $L^{1}$ data, J. Inequal. Pure Appl. Math. 6 (2005), no. 2, 1-20.

[4] A. Benkirane and J. Bennouna, Existence of entropy solutions for some nonlinear problems in Orlicz spaces, Abstr. Appl. Anal. 7 (2002), no. 2, 85-102.

[5] _ Existence of renormalized solutions for some elliptic problems involving derivatives of nonlinear terms in Orlicz spaces, Partial differential equations, Lecture Notes in Pure and Appl. Math., vol. 229, Dekker, New York, 2002, pp. 125-138.

[6] Existence and uniqueness of solution of unilateral problems with $L^{1}$-data in Orlicz spaces, Ital. J. Pure Appl. Math. 16 (2004), 87-102.

[7] A. Benkirane and A. Elmahi, A strongly nonlinear elliptic equation having natural growth terms and $L^{1}$ data, Nonlinear Anal. 39 (2000), no. 4, 403-411. 
[8] L. Boccardo, D. Giachetti, J. I. Diaz, and F. Murat, Existence and regularity of renormalized solutions for some elliptic problems involving derivatives of nonlinear terms, J. Differential Equations 106 (1993), no. 2, 215-237.

[9] G. Dal Maso, F. Murat, L. Orsina, and A. Prignet, Renormalized solutions of elliptic equations with general measure data, Ann. Scuola Norm. Sup. Pisa Cl. Sci. (4) 28 (1999), no. 4, 741-808.

[10] R. J. DiPerna and P.-L. Lions, On the Cauchy problem for Boltzmann equations: Global existence and weak stability, Ann. of Math. (2) 130 (1989), no. 2, 321-366.

[11] J.-P. Gossez, Nonlinear elliptic boundary value problems for equations with rapidly (or slowly) increasing coefficients, Trans. Amer. Math. Soc. 190 (1974), 163-205.

[12] $\_$Some approximation properties in Orlicz-Sobolev spaces, Studia Math. 74 (1982), no. 1, $17-24$.

[13] J.-P. Gossez and V. Mustonen, Variational inequalities in Orlicz-Sobolev spaces, Nonlinear Anal. 11 (1987), no. 3, 379-392.

[14] M. A. Krasnosel'skii and Ja. B. Rutickii, Convex functions and Orlicz spaces, P. Noordhoff Ltd., Groningen, 1961.

[15] J.-M. Rakotoson, Uniqueness of renormalized solutions in a T-set for the $L^{1}$-data problem and the link between various formulations, Indiana Univ. Math. J. 43 (1994), no. 2, 685-702.

[16] J.-M. Rakotoson and R. Temam, Relative rearrangement in quasilinear elliptic variational inequalities, Indiana Univ. Math. J. 36 (1987), no. 4, 757-810. 